\title{
Psychosocial therapies for the adjunctive treatment of bipolar disorder in adults: network meta-analysis
}

\author{
Mary Lou Chatterton, Emily Stockings, Michael Berk, Jan J. Barendregt, Rob Carter
}

and Cathrine Mihalopoulos

\section{Background}

Few trials have compared psychosocial therapies for people with bipolar affective disorder, and conventional metaanalyses provided limited comparisons between therapies.

\begin{abstract}
Aims
To combine evidence for the efficacy of psychosocial interventions used as adjunctive treatment of bipolar disorder in adults, using network meta-analysis (NMA).
\end{abstract}

\section{Method}

Systematic review identified studies and NMA was used to pool data on relapse to mania or depression, medication adherence, and symptom scales for mania, depression and Global Assessment of Functioning (GAF).

\section{Results}

Carer-focused interventions significantly reduced the risk of depressive or manic relapse. Psychoeducation alone and in combination with cognitive-behavioural therapy (CBT) significantly reduced medication non-adherence. Psychoeducation plus CBT significantly reduced manic symptoms and increased GAF. No intervention was associated with a significant reduction in depression symptom scale scores.

\section{Conclusions}

Only interventions for family members affected relapse rates. Psychoeducation plus CBT reduced medication nonadherence, improved mania symptoms and GAF. Novel methods for addressing depressive symptoms are required.

\section{Copyright and usage}

(c) The Royal College of Psychiatrists 2017.
Bipolar affective disorder is a chronic, severe mental disorder characterised by acute episodes of depressed, manic and mixed mood states. ${ }^{1,2}$ Globally bipolar disorder affects $0.7 \%$ of the adult population, ${ }^{3}$ and is associated with significant disability and cost owing to the severity and chronicity of the condition. ${ }^{4,5}$ In 2010 the disorder accounted for $1.3 \%$ of years of life lost to disability globally, and was the fifth leading cause of disability among mental and substance use disorders. ${ }^{4}$ Although pharmacotherapy is the recommended first-line therapy for manic, depressive and residual states, ${ }^{6}$ medication adherence is typically poor, ${ }^{7}$ relapse rates are high, ${ }^{8}$ and full remission is rare. ${ }^{1}$ Psychosocial therapies are recommended as an adjunctive treatment to medications in many guidelines, ${ }^{9-11}$ to reduce relapse to acute depression or mania, potentially through improved medication adherence, identification of early warning signs, self-management and family communication. $^{12}$ These psychosocial therapies have been evaluated through randomised controlled trials (RCTs) and meta-analyses with mixed results. Previous systematic reviews and meta-analyses were based on small numbers of studies, ${ }^{13,14}$ were limited to particular interventions (e.g. CBT only), ${ }^{15}$ did not cover key peripheral indicators of recovery and did not adjust for study characteristics such as the control groups. ${ }^{16}$ The most comprehensive review and meta-analysis recently published produced significant positive effects for some psychosocial interventions; however, multiple analyses were undertaken, several of which included only one study and did not address study quality. ${ }^{17,18}$ Importantly, the study did not use meta-regression to examine factors that might have influenced intervention efficacy. The purpose of our systematic review and network meta-analysis (NMA) was to address these previous limitations by employing the NMA approach to examine the efficacy of psychosocial interventions used for the adjunctive treatment of bipolar disorder in adults on relapse rates, depressive and manic symptoms, global functioning and medication adherence.
Meta-regression techniques were used to identify factors related to the efficacy of this group of therapies. Unlike standard metaanalysis that can compare only two treatments at a time, NMA (also called mixed treatment comparisons) can assess the relative effectiveness of several interventions simultaneously even when the treatments have not been directly compared. It combines the evidence from studies directly comparing interventions (RCTs) with indirect information (comparisons made between RCTs through a common comparator such as treatment as usual). ${ }^{19-21}$ Using NMA addresses the issue of multiple testing found in previous meta-analyses as well as providing additional information in the form of indirect comparisons. The hypothesis was that network meta-analysis would improve the rigour of the results.

\section{Method}

Electronic peer-reviewed databases including Medline, PsycINFO and the Cochrane Library of Systematic Reviews accessed through EBSCOhost, as well as the clinical trial registries ClinicalTrials.gov, EU Clinical Trials Register, ISRCTN Registry, WHO International Clinical Trials Registry Platform and the Australian New Zealand Clinical Trial Registry were searched. We used search terms pertaining to bipolar disorder (e.g. bipolar disorder, cyclothymic personality) and psychotherapy (e.g. psychotherapy, behaviour therapy, brief psychotherapy, client centred therapy, cognitive behaviour therapy, emotion focused therapy, group psychotherapy, interpersonal psychotherapy, narrative therapy, psychotherapeutic counselling, solution focused therapy). Searches were limited to studies conducted among humans and published in the English language from inception of the databases to 31 January 2016. Full details of the search strategy are provided in online Table DS1. A review protocol was registered with the 
International Prospective Register of Systematic Reviews (PROSPERO), registration number 2015:CRD42015016975. All identified publications were reviewed independently by two authors (E.S. and M.L.C.) against the selection criteria. Any discrepancies in the selection of articles were discussed among these authors to arrive at the final list of included studies.

We included randomised controlled trials that tested a psychosocial intervention against any comparator for the treatment of bipolar disorder (including types 1 and 2 disorder and cyclothymia in the acute or maintenance phase) among adults aged at least 18 years who were receiving regular mood-stabilising medication at intake. We included any intervention that comprised a psychosocial strategy alongside pharmacological treatment with the primary aim of improving bipolar disorder outcomes. We excluded studies where the sample age range included those younger than 18 years; where part or all of the sample were not receiving regular mood-stabilising medication; where the authors did not report outcomes related to changes in bipolar disorder outcomes; or where data were reported in a non-usable format (e.g. graphically) and usable data could not be obtained from the study authors. The rationale for excluding studies with people less than 18 years old related to the atypical presentation of bipolar disorder in youth, since it is unclear if a bipolar disorder diagnosis in young people is the same as in adults. ${ }^{22}$ Studies that evaluated collaborative care were excluded since this approach not only involved psychosocial interventions but also changed the delivery of care to study participants.

\section{Measures of treatment effect}

All outcomes were derived from the primary end-point of each study or the longest follow-up time point. We extracted the number of participants meeting criteria for a manic or depressive episode (pooled) at follow-up as the primary outcome of relapse. Methods used to assess relapse included standardised clinical interviews yielding DSM or ICD diagnoses, predetermined cut-off points on depression or mania symptom scales, or admission to hospital. Intervention efficacy for the pooled outcome of depressive or manic relapse was assessed using a risk ratio (RR). We extracted the means and standard deviations of participants' scores on reliable and valid symptom rating scales for depression and mania, where lower scores equated to lower levels of symptoms. We also extracted means and standard deviations of participants' scores on reliable and valid measures of global functioning such as the Personal and Social Performance Scale and the Global Assessment of Functioning (GAF) scale, ${ }^{23}$ where higher scores equated to better functioning. In instances where the direction of a scale was reversed (higher scores equated to poorer functioning) we subtracted the mean from the maximum possible score on the scale if that was available, and if not multiplied the mean values by -1 to ensure all outcomes were in the same direction. ${ }^{24}$ Intervention efficacy was determined by calculation of a standardised mean difference (SMD) on these scales (measured using Hedges' $g$ ). We extracted the number of participants classified as being adherent to their regular psychiatric medication schedule, based on serum mood stabiliser concentrations above a pre-specified cut-off level or based on patient self-report (e.g. taking medication on at least $50 \%$ of the days since the preceding assessment) or reports from carers, family members or health professionals. Medication adherence was assessed using a risk ratio.

\section{Risk of bias}

Risk of bias was examined using the Cochrane Collaboration tool and techniques for assessing risk of bias. ${ }^{24}$ Two authors (M.L.C. and E.S.) independently examined each study and rated it as low risk (score 3), high risk (score 1) or 'risk unable to be determined' (score 2) for each domain. A total quality score was calculated as the sum of all components. A quality index with a value between 0 and 1 was calculated for each study by dividing the total quality score by the highest scoring study in the group. This index was then used in the quality effects meta-analysis described below.

\section{Data extraction}

Data were extracted independently by two of the authors (M.L.C. and E.S.). Each extracted data for half the studies and then cross-checked the entries for errors and consistency. Where studies reported outcomes for particular subgroups of the sample rather than for the control and intervention groups at an aggregate level, we calculated these values using the pooled variance and weighted mean. In instances where usable data were not reported in the manuscript, attempts were made to contact study authors for unpublished data.

\section{Statistical analysis}

Each of the five outcomes evaluated required a separate network meta-analysis which was conducted using MetaXL version 5.1, an Excel add-in developed by EpiGear International Pty Ltd. ${ }^{25}$ MetaXL implements the generalised pairwise modelling framework for network meta-analysis; this makes systematic indirect estimates of the input studies, followed by meta-analysis of all interventions compared with the common control. ${ }^{25}$ Whereas most meta-analyses use a random effects model, the primary analyses for this meta-analysis employed the inverse variance heterogeneity (IVhet) model, which uses the inverse variance study weights with a quasi-likelihood based variance structure for the pooled estimate to manage the potential underestimation of the statistical error due to heterogeneity. ${ }^{26}$ We consider this superior to the random effects model because it addresses the known issue of underestimation of the statistical error with the random effects model. ${ }^{27}$ A quality effects model was used as a sensitivity analysis since it favours larger and higher-quality trials and produces a conservative confidence interval for the pooled point estimate. ${ }^{28}$ Effect sizes (Hedges' $g$ ) were specified as 0.2 small, 0.5 medium and 0.8 large. ${ }^{29}$

The assumption of transitivity, that the direct and indirect evidence are in agreement, underpins NMA. ${ }^{19}$ Consistency, a statistical measure of transitivity, was assessed through computation of the $H$ statistic which describes the relative excess in heterogeneity (Cochrane's $Q$ ) over its degrees of freedom. ${ }^{25}$ To explain any further causes of heterogeneity on the basis of study characteristics and characteristics of the intervention, we conducted weighted linear regression models using the Stata 'regress' command. Study-level weights were obtained from the IVhet meta-analyses, and were included in the regressions using the analytical weights option. Separate weighted regressions were conducted where the overall meta-analysis produced a significant result at the primary outcome time-point within each study, and thus were conducted for relapse rates, adherence rates, manic symptoms and GAF. Ratio data (including relapse rates and adherence rates) were natural log-transformed prior to analysis. We selected factors for the regression analysis a priori based on published research. The analysis examined the effect of intervention facilitator (clinician - psychiatrist or psychologist $v$. trained therapist); intervention delivery format (group $v$. individually delivered); total intervention exposure time (in minutes); follow-up time-point (in months); any psychiatric comorbidity at baseline (anxiety, schizophrenia, suicidal ideation, 
antisocial personality disorder, substance use disorder or borderline personality disorder); phase of illness at baseline (euthymic $v$. acute); and bipolar disorder type at baseline (type 1 only $v$. types 1 and 2). The adjusted $R^{2}$ index was employed to quantify goodness-of-fit for each model. Statistical significance for all analyses was set at $P<0.05$.

\section{Results}

We identified 176 studies as potentially appropriate for inclusion in the meta-analysis (Fig. 1). There was $93 \%$ agreement between reviewers $(\kappa=0.84, P<0.05)$ for full-text articles meeting the inclusion criteria. Once data were extracted, 45 publications from 41 unique trials were suitable for the pooled analyses. ${ }^{30-74}$ Thirtyeight studies were excluded owing to inappropriate study designs; 31 were excluded because of lack of appropriate results for the pooled analyses, such as group means and standard deviations at study end-points. Three trials evaluated interpersonal and social rhythm therapy (IPSRT) but only one provided outcome data that could be used in the pooled analyses, and this trial was conducted in people with type 2 bipolar disorder who were not taking medication at study entry; ${ }^{75}$ it was therefore determined not to be a trial of adjunctive therapy and excluded. A single attempt was made to contact 11 study authors where insufficient or unusable data were reported in the manuscript. Two responses were received, and one author provided additional data. However, that study was subsequently deemed not to fit the inclusion criteria as noted above and was excluded. ${ }^{75}$

The 41 trials analysed in the meta-analysis contained data from a combined total of 3119 individuals and are described in

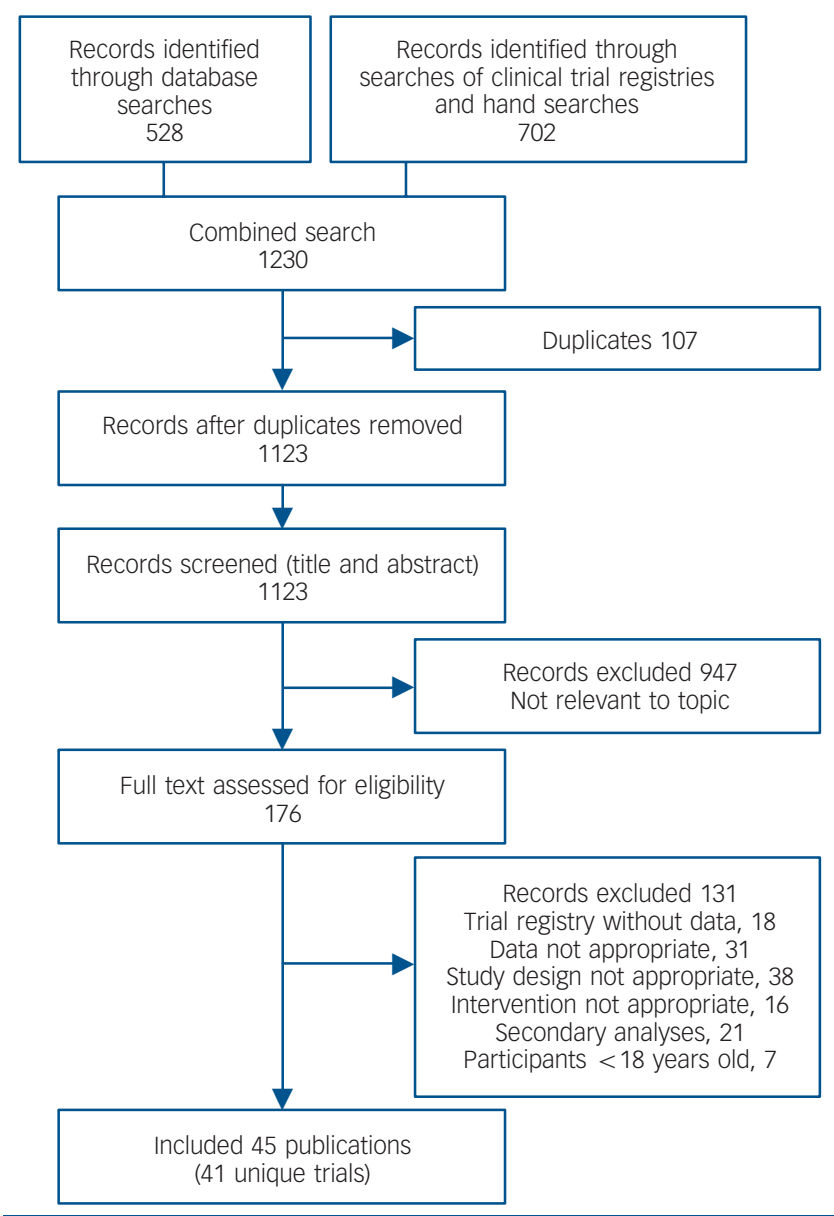

Fig. 1 PRISMA flow chart of the study selection process online Table DS2. Gender was reasonably balanced with 57\% of participants being female, and ages ranged from 18 years to 77 years (mean 38.8, s.d. $=10.7$ ). Most studies recruited patients diagnosed with the broad inclusion criteria of bipolar disorder according to DSM criteria, which include type 1, 2 or cyclothymic disorder (33 trials, $n=2620$ )..$^{30,33-48,52,54,55,60,61,63-66,68-74}$ Nearly half of the studies required patients to be euthymic at intake (20 trials, $n=1262) .^{30,33,35,37,38,40-42,44-46,48-50,55,59,63,69,73,74}$

The majority of trials evaluated CBT (16 trials) and psychoeducation (12 trials) (Table 1). Another group of four trials clearly described the intervention as a combination of psychoeducation and CBT and were analysed as a separate subgroup. Three studies were included that evaluated family-focused therapy (FFT). A separate category of carer-focused interventions included data from five evaluations. A novel study evaluating psychoeducation combined with a mobile telephone application, Personalized Real-time Intervention for Stabilizing Mood (PRISM), was analysed as a separate group. The outcome measures that could be combined in meta-analysis varied across studies, with depression symptom scales being the most common outcome measure available from 25 trials, relapse (depression or mania) assessed in 24 trials, mania rating scales reported in 18, GAF scores from 14 and medication adherence from 11. Additional details of the studies used for the meta-analysis are presented in online Table DS2. The most common comparator was treatment as usual (TAU); eight trials contained active comparators. ${ }^{35,38,52,55,60,62,72,74}$

We assessed all included studies for risk of bias (see online Figs DS1 and DS2). Of the 41 included trials, reporting of random sequence generation and allocation concealment was mostly absent, and as such most studies were rated as unclear for these domains (27 and 21 studies respectively). Most studies either did not report masking of participants or reported that participants were actively aware of their treatment allocation, and thus 26 and 10 studies were rated respectively as unclear or at high risk of bias; however, masking of treatment allocation is acknowledged as being inherently difficult in studies of behavioural interventions. ${ }^{76}$ Masking of outcome assessors was mostly done, and most trials (24) were rated as low risk for this domain. Similarly, most studies (24) were rated as low risk for attrition bias, as authors primarily conducted intention-to-treat analyses and reported low and equal attrition between treatment conditions. Although just over half of studies were classified as having a low risk of bias due to selective reporting (21 studies), more than a third were classified as high risk for this domain (15 studies), primarily where raw mean scores and standard deviations on symptom screening scales for depression and mania were not reported, or where raw numbers of participants meeting criteria for relapse were not reported or only displayed graphically. Most studies were classified as having a low risk of bias due to any other cause (31 studies); however, 8 studies were rated as having high risk of bias, mostly where the intervention and control groups were not equivalent on key measures of depression and mania at baseline (e.g. significant differences in mean scores on symptom screening scales).

The network diagram for the outcome of pooled manic or depressive relapse (online Fig. DS3) contained seven nodes: five psychosocial interventions and two control conditions. The carer-focused interventions were significantly more efficacious than TAU on the outcome of relapse with the IVhet model as shown in the forest plot (Fig. 2(a)): RR $=0.61,95 \%$ CI $0.44-$ 0.86 ). Only two trials of carer interventions (70 participants in total) were used in this analysis, but the quality of both was high. Family-focused therapy, psychoeducation and CBT were also associated with reduced risk ratios for relapse $v$. the control condition of TAU; however, these were not significant with the 


\section{Cognitive-behavioural therapy}

In CBT therapists aim to work collaboratively with patients to understand the link between thoughts, feelings and behaviours, and to identify and modify unhelpful thinking patterns, underlying assumptions and idiosyncratic cognitive schemata about the self, others and the world. This category also includes 'third wave' CBT such as mindfulness exercises ${ }^{82}$

\section{Psychoeducation}

Psychoeducation aims to provide information on the disorder and its treatment, as well as the social and family consequences of the disorder

Ball et al (2006) $)^{30}$

Cochran (1984) $)^{34}$

Costa et al $(2011)^{37}$

Gomes et al (2011) $)^{42}$

Jones et al $(2015)^{46}$

Kirk \& Gumley (2014) ${ }^{47}$

Lahera et al $(2013)^{48}$

Lam et al $(2000)^{49}$

Lam et al (2003), ${ }^{50}(2005)^{83}$

Meyer \& Hautzinger (2012) ${ }^{255}$

Perich et al $(2013)^{59}$

Schmitz et al (2002) $)^{65}$

Scott et al $(2001)^{66}$

Scott et al (2006) ${ }^{67}$

Weiss et al (2009) 72

Williams et al $(2008)^{73}$

\section{Cardoso et al (2015) 32}

Castle et al $(2010)^{33}$

Colom et al (2003), ${ }^{35}$ (2009) $^{\text {a } 36}$

Dogan \& Sabanciogullari (2003)

D'Souza et al (2010) ${ }^{40}$

Eker \& Harkin $(2012)^{41}$

Javadpour et al $(2013)^{45}$

Lin et al $(2015)^{53}$

Perry et al (1999) ${ }^{61}$

Sajatovic et al (2009) ${ }^{64}$

Simon et al $(2005)^{68}$

Smith et al $(2011)^{69}$

Gonzalez-Isasi et al (2010), ${ }^{43}(2014)^{44}$

Lauder et al (2015) $)^{\text {a } 52}$

Van Dijk et al (2013)

Zaretsky et al (2008) ${ }^{\text {a }} 74$

Depp et al (2015) $)^{\text {a } 38}$

Psychoeducation and Personalized Real-time Intervention for Stabilizing Mood

Family-focused therapy

Family-focused therapy is based on the premise that aversive family environments affect the course of bipolar disorder. Treatment consists of psychoeducation, communication enhancement training and problem-solving training. These sessions included the person with bipolar disorder and family members to address the family dynamics that may affect the course of the disorder

Carer-focused interventions

Carer-focused interventions used techniques such as psychoeducation, but included only the carer or family member. Note that the data extracted for the meta-analysis was based on the outcomes for the person with disorder not the carer/family member
Miklowitz et al (2000), ${ }^{56}(2003)^{57}$ Miller et al (2004) 58 Rea et al (2003) ${ }^{\text {a }} 62$
$A, D, G A F, M, R$

A, R

$D, M$

$\mathrm{R}$

D, GAF, R

D, GAF

$D, G A F, M$

$D, G A F, M, R$

$A, D, M, R$

$D, G A F, M, R$

$D, M$

A

D, GAF, R

D, M D

D, M

$D, M, R$

A, $R$

A, D, GAF

$D, M, R$

A

$\mathrm{R}$

D, M, R

D, GAF, M

$D, G A F, M, R$

$D, G A F, M$

$D, M, R$

A, R

D, GAF, M

D, R

A, R

$\begin{array}{ll}\text { Bordbar et al (2009) } & \text { R } \\ \text { Madigan et al (2012) } & \text { GAF } \\ \text { Perlick et al }(2010)^{\text {a }} \text { 60 } & \text { D, M } \\ \text { Reinares et al (2008) }{ }^{63} & \text { A, R } \\ \text { Van Gent \& Zwart (1991) } & \text { A, GAF }\end{array}$

A, adherence $\mathrm{CBT}$, cognitive-behavioural therapy: $\mathrm{D}$, depression symptom scales; GAF, Global Assessment of Functioning: $M$, mania rating scales; $\mathrm{R}$, relapse.

A, adherence; CBT, cognitive-behavioural th

a. Studies with an active comparator group.
b. Studies with more than one treatment group.

IVhet model or QE models (forest plots are shown in online Figs DS8-17). The network of interventions with depression symptom scale score outcomes (online Fig. DS4) included six psychosocial interventions and three control conditions. One trial compared a carer-focused intervention with an attention control condition, which meant it was disconnected from the network and therefore could not be analysed in the network meta-analysis. ${ }^{60}$ The SMD results presented in Fig. 2(b) indicate that although three of the psychosocial interventions (psychoeducation plus CBT, FFT and psychoeducation) and one of the control conditions (group drug counselling) decreased depression symptom scores compared with TAU, none had a significant effect. The combination of psychoeducation and PRISM significantly increased depression scores compared with TAU.
The network of interventions providing mania symptom scale score outcomes was similar to the depression network with the exception of lacking the FFT $v$. TAU branch (online Fig. DS5). The carer-focused intervention and attention control node was disconnected and thus could not be analysed as part of the network. Figure 2(c) shows that the combination of psychoeducation and CBT had a significant, large effect on reducing manic symptoms v. TAU (SMD $=-0.95,95 \% \mathrm{CI}-1.47$ to -0.43 ). This intervention was also superior to all other interventions included. The effect of psychosocial therapies on the risk of non-adherence are shown in Fig. 2(d) and the network diagram in online Fig. DS6. People who received the combination of psychoeducation and CBT were significantly less likely to report non-adherence to their bipolar medications than those receiving TAU $(\mathrm{RR}=0.14,95 \%$ 
(a)

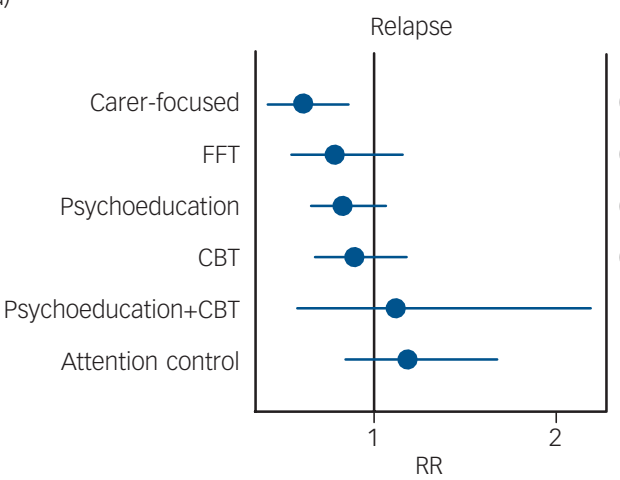

(c)

Manic symptoms

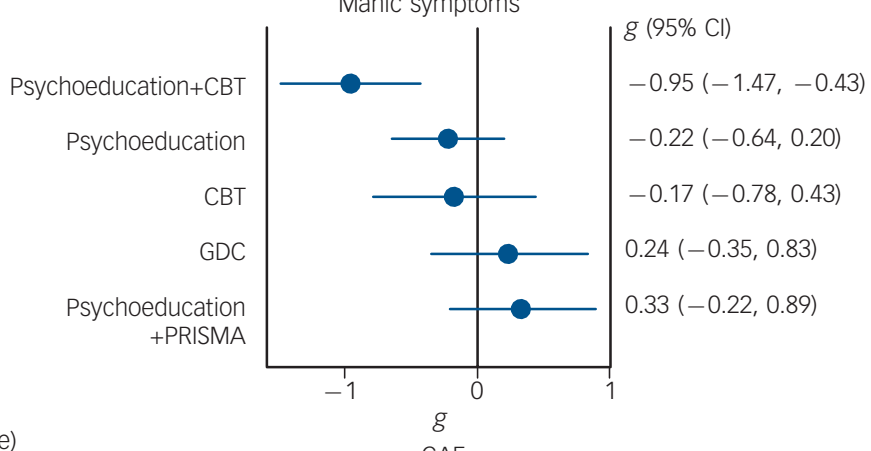

GAF

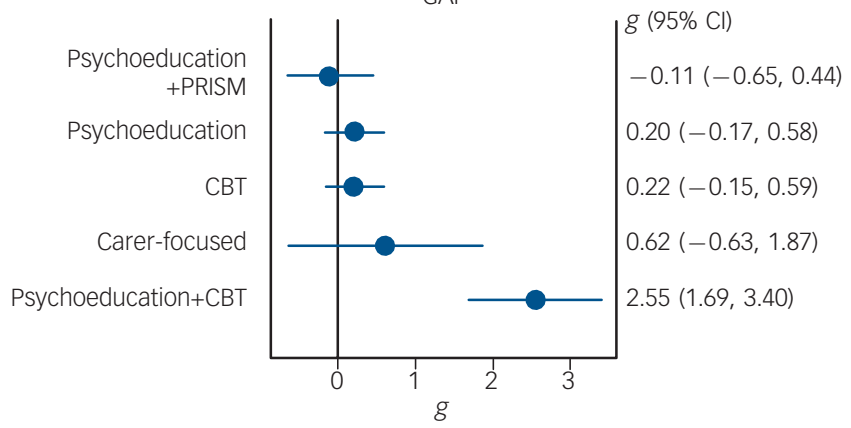

(b)

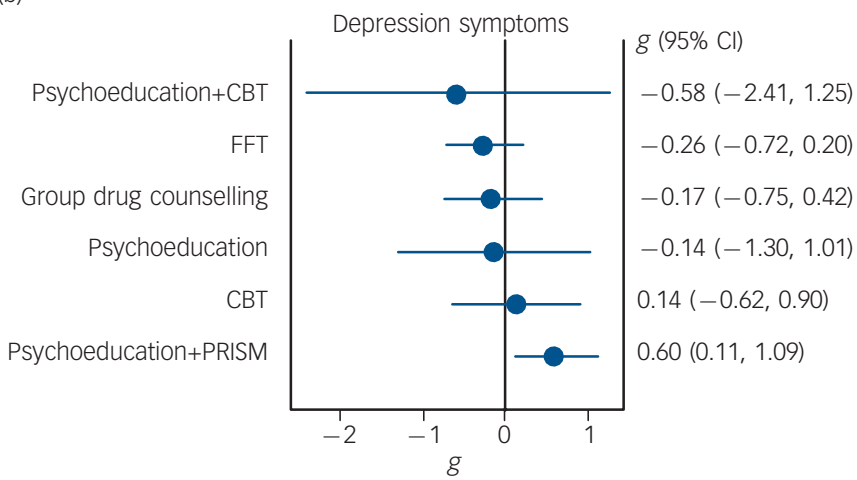

(d)

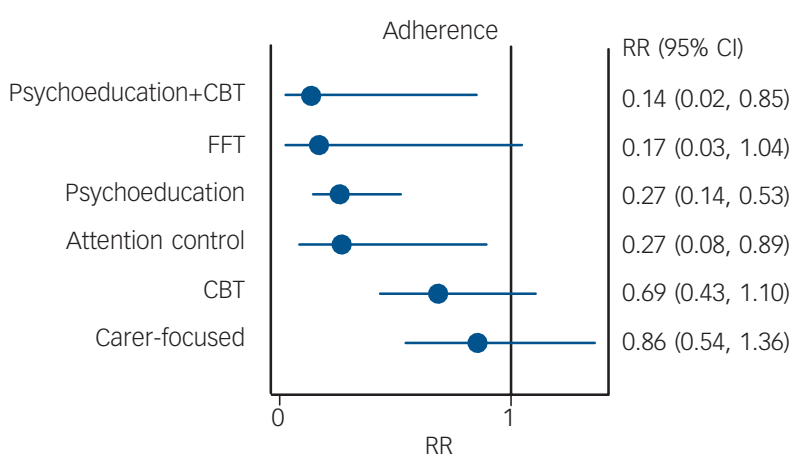

Fig. 2 Forest plots of network meta-analysis results (all $v$. treatment as usual). (a) Relapse: all treatments; (b) all depression symptoms; (c) all mania symptoms; (d) adherence to medication; (e) Global Assessment of Functioning. CBT, cognitive-behavioural therapy; FFT, family-focused therapy; GAF, Global Assessment of Functioning; GDC, group drug counselling; RR, risk ratio

\section{Table 2 Weighted regressions examining factors related to intervention efficacy at the longest follow-up assessment}

\begin{tabular}{|c|c|c|c|c|c|c|c|c|c|c|c|c|}
\hline & \multicolumn{3}{|c|}{ Relapse rate } & \multicolumn{3}{|c|}{ Adherence rate } & \multicolumn{3}{|c|}{ Manic symptoms } & \multicolumn{3}{|c|}{ GAF } \\
\hline & $\begin{array}{c}t \\
\text { (d.f. }=25)\end{array}$ & $P$ & $\begin{array}{l}\text { Adj. } R^{2} \\
(\%)\end{array}$ & $\begin{array}{c}t \\
\text { (d.f. }=12)\end{array}$ & $P$ & $\begin{array}{l}\text { Adj. } R^{2} \\
(\%)\end{array}$ & $\begin{array}{c}t \\
\text { (d.f. }=18)\end{array}$ & $P$ & $\begin{array}{l}\text { Adj. } R^{2} \\
(\%)\end{array}$ & $\begin{array}{c}t \\
\text { (d.f. }=15)\end{array}$ & $P$ & $\begin{array}{l}\text { Adj. } R^{2} \\
(\%)\end{array}$ \\
\hline Facilitator type ${ }^{a}$ & 1.85 & 0.07 & 9.23 & 0.41 & 0.69 & -8.15 & -0.70 & 0.49 & -3.08 & 1.02 & 0.33 & 0.30 \\
\hline $\begin{array}{l}\text { Intervention } \\
\text { delivery format }{ }^{\mathrm{b}} \\
\text { Total intervention }\end{array}$ & -1.64 & 0.11 & 6.63 & 0.09 & 0.93 & -9.92 & -0.61 & 0.55 & -3.83 & 1.18 & 0.26 & 2.77 \\
\hline exposure & 0.84 & 0.42 & -1.71 & -1.50 & 0.18 & 13.38 & -0.77 & 0.46 & -3.26 & 1.02 & 0.34 & -0.49 \\
\hline $\begin{array}{l}\text { Follow-up length }{ }^{d} \\
\text { Psychiatric }\end{array}$ & 0.83 & 0.41 & -1.28 & -1.56 & 0.15 & 11.46 & -2.63 & 0.02 & $25.83^{*}$ & 5.19 & $<0.01$ & $64.98^{*}$ \\
\hline comorbidity $^{\mathrm{e}}$ & 2.38 & 0.03 & $16.32^{\star}$ & 0.10 & 0.92 & -9.90 & 1.59 & 0.13 & 8.24 & -0.95 & 0.36 & -0.66 \\
\hline Phase of illness ${ }^{f}$ & 0.90 & 0.38 & -0.08 & 1.29 & 0.23 & 5.67 & 0.22 & 0.83 & -5.93 & 0.31 & 0.76 & -6.89 \\
\hline Disorder type ${ }^{g}$ & 1.64 & 0.11 & 6.61 & -1.40 & 0.19 & 8.02 & 0.92 & 0.38 & -0.95 & -3.03 & 0.01 & $36.95^{\star}$ \\
\hline \multicolumn{13}{|c|}{$\begin{array}{l}\text { Adj., adjusted; GAF, Global Assessment of Functioning. } \\
\text { a. Clinician (psychiatrist or psychologist) } v \text {. trained therapist. } \\
\text { b. Group } v \text {. individually delivered. } \\
\text { c. Total intervention exposure in minutes. } \\
\text { d. Length of final follow-up in months. } \\
\text { e. Patients with comorbid psychiatric disorders (anxiety, schizophrenia, suicidal ideation, antisocial personality disorder, substance use disorder or borderline personality disorder) } \\
\text { at intake } v \text {. those without. } \\
\text { f. Euthymic state at intake } v \text {. acute phase. } \\
\text { g. Type } 1 \text { bipolar affective disorder } v \text {. types } 1 \text { and } 2 \text {. } \\
\star P<0.05 \text {. }\end{array}$} \\
\hline
\end{tabular}


CI 0.02-0.85). Psychoeducation alone was also associated with a significantly lower risk of non-adherence with bipolar medications $(\mathrm{RR}=0.27,95 \%$ CI $0.14-0.53)$ as was the attention control condition ( $\mathrm{RR}=0.27,95 \% \mathrm{CI} 0.08-0.89)$. The network of interventions with GAF outcomes contained six nodes (online Fig. DS7). Psychoeducation and CBT had a significant large effect on GAF scale scores compared with TAU (SMD $=2.55,95 \% \mathrm{CI}$ 1.69-3.40), as shown in Fig. 2(e). The network meta-analysis results for the depression, mania and GAF scales did not differ with the quality effects models (see online Figs DS8-17 for results using the alternative models). The weighted average $H$ statistic for all the networks in this analysis was less than 3 , indicating minimal inconsistency in treatment effects. The specific $H$ values using the inverse variance heterogeneity model were relapse 1.06, depression symptom scores 2.14 , mania symptom scores 1.26 , adherence 1 , GAF 1.11 .

\section{Meta-regression}

Weighted regressions revealed that the risk of relapse was higher in studies where samples comprised participants with any comorbid psychiatric disorder at baseline, compared with studies where participants were required to have no existing comorbid psychiatric disorder to meet inclusion criteria: $t(25)=2.38$, $P=0.03$, adjusted $R^{2}=16.3 \%$; Table 2, online Fig. DS18). No factor was significantly associated with adherence rates. For symptoms of mania and GAF scores, studies with longer follow-up data collection had larger effect sizes than those collecting data only in the mid or short term: $t(18)=2.63, P=0.02$, adjusted $R^{2}=25.8 \%$ for mania and $t(15)=5.19, P<0.01$, adjusted $R^{2}=65.0 \%$ for GAF scores (Table 2, online Figs DS19 and DS20). Additionally, effect sizes for improvements in GAF scores were smaller in studies where samples comprised participants diagnosed with type 1 and 2 disorder, compared with studies where samples were limited to people with type 1 only: $t(15)=-3.03, P=0.01$, adjusted $R^{2}=36.9 \%$ (Table 2, online Fig. DS21).

\section{Discussion}

To our knowledge this is the first network meta-analysis of psychosocial interventions used as adjunctive treatment for bipolar disorder, and the first meta-analysis to report pooled results for the outcomes of medication adherence and GAF for these interventions in people with this disorder. The study method addressed limitations in prior meta-analyses. The use of the IVhet and QE models as well as the addition of meta-regression increased the robustness of the findings. The results suggest that many psychosocial interventions lack high-quality evidence to support their efficacy as an adjunctive treatment to improve outcomes for people with bipolar disorder. Only the carer-focused interventions were associated with a significant reduction in the risk of relapse $v$. TAU. The effect for the carer interventions translates into a $39 \%$ relative risk reduction in relapse compared with TAU. However, the use of TAU as a control tends to inflate effect sizes compared with the use of active controls. ${ }^{16}$ It is also important to note that only five RCTs evaluating carer-focused interventions could be included in this systematic review, suggesting a need for additional trials of this therapy in particular. Psychoeducation (delivered individually or in a group) as well as FFT and CBT showed trends toward improvement in the risk ratio but these results were non-significant under both IVhet and QE models. The lack of a significant effect on the risk of relapse for CBT was similar to the non-significant findings by Lynch et al. ${ }^{15}$ However, our analysis included five additional RCTs and was based on direct and indirect comparisons providing additional strength to the results. These results contrast with those of Oud et al, ${ }^{18}$ who found significant effects on depressive and manic relapses for group psychological interventions as well as for any relapse for individual psychological interventions at post-treatment and follow-up compared with TAU. The differences are probably due to differences in methodology (standard meta-analysis $v$. network) and the grouping of studies, since Oud et al had more groups with smaller numbers of studies per analysis.

None of the psychosocial interventions was found to have a significant effect in reducing depression symptom scale scores. Our analysis was based on the longest follow-up point and was similar to that of Oud et al, who showed that individual psychological interventions did not significantly reduce depression symptoms at follow-up assessments. ${ }^{18}$ Depressive symptoms in bipolar disorder are poorly addressed by both existing medication and psychotherapy strategies, reflecting the largest unmet need in the disorder. ${ }^{77}$ Novel psychotherapeutic approaches to target depression and emotion regulation remain the next treatment horizon. ${ }^{78}$

The combination of psychoeducation and CBT demonstrated a significant large effect on reducing mania symptom scale scores compared with TAU as well as in comparison to all other comparators. This appears consistent with the significant but small effect that was seen in a previous meta-analysis for individual psychological interventions. ${ }^{18}$ However, other meta-analyses have not evaluated this combination of psychosocial interventions because the trials combining these two psychosocial therapies are recent, with the first trial published in 2008. Psychoeducation and the combination of psychoeducation and CBT were also found to significantly reduce the risk of medication non-adherence compared with TAU, which translates into a relative risk reduction for non-adherence of $86 \% v$. TAU. Psychoeducation alone as well as the attention control comparator were also found to have a significant relative risk reduction of $73 \%$ compared with TAU. This appears to be the first meta-analysis to report these effects, but it should be interpreted prudently since the network of trials used in this analysis was limited. The combination of psychoeducation and CBT was also found to have a significantly large improvement in GAF scores compared with TAU as well as compared with psychoeducation, CBT, carer-focused interventions and psychoeducation plus PRISM. This result should be viewed cautiously since only one direct comparison of psychoeducation and CBT compared with TAU was included in the network of 15 trials for this analysis, and the result relies heavily on the indirect comparisons within the network.

The meta-regression results indicated that the risk of relapse was higher in studies comprising samples with psychiatric comorbidities, which has been described previously. ${ }^{79,80}$ The greater improvements in mania symptoms and GAF at long-term follow-up relative to the short term may be indicative of increased efficacy of the interventions over time. The meta-regression of bipolar disorder type and GAF suggests that the psychosocial interventions have a greater benefit to global functioning in people diagnosed with type 1 bipolar disorder than for the group having a mix of type 1 and 2 diagnoses. This finding may be reflecting a similar mechanism to the higher risk of relapse among samples with psychiatric comorbidities, suggesting that psychosocial interventions may need to be tailored to specific manifestations of bipolar disorder and existing comorbidities, owing to the more severe course of illness associated with these disorders. ${ }^{81}$

\section{Limitations and strengths}

This analysis was limited by the number of RCTs available for inclusion and the data that could be pooled. Numerous potentially eligible studies could not be included owing to the lack of reporting of extractable data, including interventions testing 
IPSRT. The variety of outcomes assessed and the methods for assessing each outcome also limited the use of specific studies in these analyses. Consistency in the use of outcome measures would vastly improve the methodological rigour of the evaluations. The analysis was also limited by potential differences in the TAU control condition used in many of the trials. Although we have considered TAU to be a homogeneous control condition, there is the potential for variability within TAU due to physician knowledge of diagnosis, prescribing, referrals and use of treatment guidelines. ${ }^{51,77,78}$ Given the small number of studies we were unable to undertake further analysis to explore this issue in detail.

Despite these limitations, these network meta-analyses strengthen treatment assessment and increase the precision of estimates through the combination of direct and indirect comparisons. The analyses examined the robustness of results under the inverse variance heterogeneity and quality effects models. They also included the assessment of peripheral improvements that are important to psychosocial functioning, including medication adherence and global functioning, which have not been included in previous reviews.

\section{Study implications}

Our results suggest that more research with a standard set of outcome measures similar to those used for trials of medications would greatly improve the rigour and comparability of the evaluations for future network meta-analyses. Given the differences in the resources required to deliver the interventions evaluated, additional cost-effectiveness assessments of the efficacious interventions from these network meta-analyses should be undertaken.

Our findings are important from both clinical and economic perspectives. From a clinical perspective, given the limited data available, this analysis suggests that psychosocial interventions should be offered to family members and carers since they significantly reduced risk of relapse in people with bipolar disorder. Psychoeducation should be offered to people with this disorder since the analysis found that it significantly reduced the risk of medication non-adherence. The combination of psychoeducation and CBT was also associated with significant decreases in the risk of medication non-adherence, reduced mania symptoms and improved global functioning and should be considered as an additional offering to people with bipolar disorder, particularly since it can be offered online. ${ }^{52}$ From an economic perspective these psychosocial therapies should be further evaluated to determine whether they are good value for money. Management of depression, however, remains the major unmet clinical need in the disorder.

\footnotetext{
Mary Lou Chatterton, PharmD, Deakin Health Economics, Centre for Population Health Research, Deakin University, Geelong, Victoria; Emily Stockings, PhD, National Drug and Alcohol Research Centre, University of New South Wales, Sydney, NSW; Michael Berk, PhD, Deakin University, IMPACT Strategic Research Centre, Barwon Health, Geelong, and Department of Psychiatry, Florey Institute of Neuroscience and Mental Health, and Orygen Youth Health Research Centre, University of Melbourne, Parkville, Victoria; Jan J. Barendregt, PhD, Epigear University of Melbourne, Parkville, Victoria; Jan J. Barendregt, PhD, Epigear
International Pty Ltd, Sunrise Beach, and School of Public Health, University of International Pty Ltd, Sunrise Beach, and School of Public Health, University of
Queensland, Brisbane, Queensland; Rob Carter, PhD, Cathrine Mihalopoulos, PhD, Deakin Health Economics, Centre for Population Health Research, Deakin University, Geelong, Victoria, Australia

Correspondence: Dr Mary Lou Chatterton, Deakin University, Waterfront Campus, Room D1.107, Locked Bag 20000, Geelong, VIC 3220, Australia. Email: m.chatterton@deakin.edu.au

First received 15 Aug 2016, final revision 30 Oct 2016, accepted 6 Nov 2016
}

\section{Funding}

This project was funded by the National Health and Medical Research Council (NHMRC grant APP1041131); E.S. and C.M. are supported by NHMRC Early Career Fellowships (1104600 and 1035887 respectively) and M.B. is supported by a NHMRC Senior Principal
Research Fellowship 1059660. The views expressed in this paper are solely those of the authors and do not reflect the views of the NHMRC. The funder of the study had no role in study design, data collection, data analysis, data interpretation or writing of the report.

\section{References}

1 American Psychiatric Association. Diagnostic and Statistical Manual of Mental Disorders (4th edn, text revision) (DSM-IV-TR). APA, 2000.

2 World Health Organization. The ICD-10 Classification of Mental and Behavioural DIsorders: Clinical Descriptions and Diagnostic Guidelines. WHO, 1992

3 Ferrari AJ, Baxter AJ, Whiteford HA. A systematic review of the global distribution and availability of prevalence data for bipolar disorder. $J$ Affect Disord 2011; 134: 1-13.

4 Whiteford HA, Degenhardt L, Rehm J, Baxter AJ, Ferrari AJ, Erskine HE, et al. Global burden of disease attributable to mental and substance use disorders: findings from the Global Burden of Disease Study 2010. Lancet 2013; 382 1575-86.

5 Kleine-Budde K, Touil E, Moock J, Bramesfeld A, Kawohl W, Rossler W. Cost of illness for bipolar disorder: a systematic review of the economic burden. Bipolar Disord 2014; 16: 337-53.

6 Yatham LN, Kennedy SH, Parikh SV, Schaffer A, Beaulieu S, Alda M, et al. Canadian Network for Mood and Anxiety Treatments (CANMAT) and International Society for Bipolar Disorders (ISBD) collaborative update of CANMAT guidelines for the management of patients with bipolar disorder: update 2013. Bipolar Disord 2013; 15: 1-44.

7 Arvilommi P, Suominen K, Mantere O, Leppamaki S, Valtonen $\mathrm{H}$, Isometsa E. Predictors of adherence to psychopharmacological and psychosocial treatment in bipolar I or II disorders - an 18-month prospective study. J Affect Disord 2014; 155: 110-7.

8 Gitlin MJ, Swendsen J, Heller TL, Hammen C. Relapse and impairment in bipolar disorder. Am J Psychiatry 1995; 152: 1635-40.

9 Royal Australian and New Zealand College of Psychiatrists Clinical Practice Guidelines Team for Bipolar Disorder. Australian and New Zealand clinical practice guidelines for the treatment of bipolar disorder. Aust NZ J Psychiatry 2004; 38: 280-305.

10 Yatham LN, Kennedy SH, Parikh SV, et al. Canadian Network for Mood and Anxiety Treatments (CANMAT) and International Society for Bipolar Disorders (ISBD) collaborative update of CANMAT guidelines for the management of patients with bipolar disorder: update 2013. Bipolar Disord 2013; 15: 1-44.

11 National Institute for Health and Care Excellence. Bipolar Disorder: The Assessment and Management of Bipolar Disorder in Adults, Children and Young People in Primary and Secondary Care. Clinical Guideline CG185. NICE, 2014

12 Miklowitz DJ. Psychosocial interventions for bipolar disorder: a critical review of evidence for efficacy. In Bipolar Disorder: A Clinician's Guide to Treatment Management, 2nd edn (eds LN Yatham, V Kusumakar): 575-90. Routledge, 2009

13 Scott J, Colom F, Vieta E. A meta-analysis of relapse rates with adjunctive psychological therapies compared to usual psychiatric treatment for bipolar disorders. Int Neuropsychopharmacol 2007; 10: 123-9.

14 Lam DH, Burbeck R, Wright K, Pilling S. Psychological therapies in bipolar disorder: the effect of illness history on relapse prevention - a systematic review. Bipolar Disord 2009; 11: 474-82.

15 Lynch D, Laws KR, McKenna PJ. Cognitive behavioural therapy for majo psychiatric disorder: does it really work? A meta-analytical review of well-controlled trials. Psychol Med 2010; 40: 9-24.

16 Furukawa TA, Noma $H$, Caldwell DM, Honyashiki M, Shinohara $K$, Imai $H$, et al. Waiting list may be a nocebo condition in psychotherapy trials: a contribution from network meta-analysis. Acta Psychiatr Scand 2014; 130: 181-92.

17 Jauhar S, McKenna PJ, Laws KR. NICE guidance on psychological treatments for bipolar disorder: searching for the evidence. Lancet Psychiatry 2016; 3: 386-8.

18 Oud M, Mayo-Wilson E, Braidwood R, Schulte $\mathrm{P}$, Jones $\mathrm{SH}$, Morriss R, et al. Psychological interventions for adults with bipolar disorder: systematic review and meta-analysis. Br J Psychiatry 2016; 208: 213-22.

19 Jansen JP, Fleurence R, Devine B, Itzler R, Barrett A, Hawkins N, et al. Interpreting indirect treatment comparisons and network meta-analysis for health-care decision making: report of the ISPOR Task Force on Indirect Treatment Comparisons Good Research Practices: part 1. Value Health 2011; 14: $417-28$

20 Hoaglin DC, Hawkins N, Jansen JP, Scott DA, Itzler R, Cappelleri JC, et al. Conducting indirect-treatment-comparison and network-meta-analysis studies: report of the ISPOR Task Force on Indirect Treatment Comparisons Good Research Practices: part 2. Value Health 2011; 14: 429-37. 
21 Cipriani A, Higgins JP, Geddes JR, Salanti G. Conceptual and technical challenges in network meta-analysis. Ann Intern Med 2013; 159: 130-7.

22 McClellan J, Kowatch R, Findling RL. Practice parameter for the assessment and treatment of children and adolescents with bipolar disorder. J Am Acad Child Adolesc Psychiatry 2007; 46: 107-25.

23 Endicott J, Spitzer RL, Fleiss JL, Cohen J. The global assessment scale. A procedure for measuring overall severity of psychiatric disturbance. Arch Gen Psychiatry 1976; 33: 766-71.

24 Higgins JPT, Green S. Cochrane Handbook for Systematic Reviews of Interventions Version 5.1.0. Cochrane Collaboration, 2011

25 Barendregt JJ, Doi SA. MetaXL User Guide Version 5.1. Epigear, 2016.

26 Doi SA, Barendregt JJ, Khan S, Thalib L, Williams GM. Advances in the metaanalysis of heterogeneous clinical trials. I: The inverse variance heterogeneity model. Contemp Clin Trials 2015; 45(A): 130-8.

27 Brockwell SE, Gordon IR. A comparison of statistical methods for metaanalysis. Stat Med 2001; 20: 825-40.

28 Doi SA, Barendregt JJ, Khan S, Thalib L, Williams GM. Advances in the metaanalysis of heterogeneous clinical trials. II: The quality effects model. Contemp Clin Trials 2015; 45(A): 123-9.

29 Hedges LV, Olkin I. Statistical Methods for Meta-analysis. Academic Press, 1985.

30 Ball JR, Mitchell PB, Corry JC, Skillecorn A, Smith M, Malhi GS. A randomized controlled trial of cognitive therapy for bipolar disorder: focus on long-term change. J Clin Psychiatry 2006; 67: 277-86.

31 Bordbar M, Soltanifar A, Talaei A. short-term family-focused psychoeducational program for bipolar disorder in Mashhad. Iran J Med Sci 2009; 34: 6 .

32 Cardoso T, Mondin TC, Reyes AN, Zeni CP, Souza LD, da Silva RA, et al. Biological rhythm and bipolar disorder: twelve-month follow-up of a randomized clinical trial. J Nerv Ment Dis 2015; 203: 792-7.

33 Castle D, White C, Chamberlain J, Berk M, Berk L, Lauder S, et al. Groupbased psychosocial intervention for bipolar disorder: randomised controlled trial. Br J Psychiatry 2010; 196: 383-8.

34 Cochran SD. Preventing medical noncompliance in the outpatient treatmen of bipolar affective disorders. J Consult Clin Psychol 1984; 52: 873-8.

35 Colom F, Vieta E, Martinez-Aran A, Reinares M, Goikolea JM, Benabarre A et al. A randomized trial on the efficacy of group psychoeducation in the prophylaxis of recurrences in bipolar patients whose disease is in remission. Arch Gen Psychiatry 2003; 60: 402-7.

36 Colom F, Vieta E, Sánchez-Moreno J, Palomino-Otiniano R, Reinares M, Goikolea JM, et al. Group psychoeducation for stabilised bipolar disorders: 5-year outcome of a randomised clinical trial. Br J Psychiatry 2009; 194: 260-5.

37 Costa RT, Cheniaux E, Rosaes PAL, Carvalho MR, Freire RC, Versiani M, et al. The effectiveness of cognitive behavioral group therapy in treating bipolar disorder: a randomized controlled study. Rev Bras Psiquiatr 2011; 33: 144-9.

38 Depp CA, Ceglowski J, Wang VC, Yaghouti F, Mausbach BT, Thompson WK, et al. Augmenting psychoeducation with a mobile intervention for bipolar disorder: a randomized controlled trial. J Affect Disord 2015; 174: 23-30.

39 Dogan S, Sabanciogullari S. The effects of patient education in lithium therapy on quality of life and compliance. Arch Psychiatr Nurs 2003; 17: 270-5.

40 D'Souza R, Piskulic D, Sundram S. A brief dyadic group based psychoeducation program improves relapse rates in recently remitted bipolar disorder: a pilot randomised controlled trial. J Affect Disord 2010; 120: 272-6.

41 Eker F, Harkin S. Effectiveness of six-week psychoeducation program on adherence of patients with bipolar affective disorder. J Affect Disord 2012; 138: 409-16.

42 Gomes BC, Abreu LN, Brietzke E, Caetano SC, Kleinman A, Nery FG, et al. A randomized controlled trial of cognitive behavioral group therapy for bipolar disorder. Psychother Psychosom 2011; 80: 144-50.

43 Isasi AG, Echeburua E, Liminana JM, Gonzalez-Pinto A. How effective is a psychological intervention program for patients with refractory bipolar disorder? A randomized controlled trial. J Affect Disord 2010; 126: 80-7.

44 Gonzalez Isasi A, Echeburua E, Liminana JM, Gonzalez-Pinto A Psychoeducation and cognitive-behavioral therapy for patients with refractory bipolar disorder: a 5-year controlled clinical trial. Eur Psychiatry 2014; 29: 134-41.

45 Javadpour A, Hedayati A, Dehbozorgi GR, Azizi A. The impact of a simple individual psycho-education program on quality of life, rate of relapse and medication adherence in bipolar disorder patients. Asian J Psychiatry 2013; 6: $208-13$
46 Jones SH, Smith G, Mulligan LD, Lobban F, Law H, Dunn G, et al. Recoveryfocused cognitive-behavioural therapy for recent-onset bipolar disorder: randomised controlled pilot trial. Br J Psychiatry 2015; 206: 58-66.

47 Kirk JD, Gumley A. Bipolar intervention study: cognitive interpersonal therapy (BISCIT) (NCT01315028). ClinicalTrials.gov, 2014

48 Lahera G, Benito A, Montes JM, Fernandez-Liria A, Olbert CM, Penn DL. Social cognition and interaction training (SCIT) for outpatients with bipolar disorder. J Affect Disord 2013; 146: 132-6.

49 Lam DH, Bright J, Jones S, Hayward P, Schuck N, Chisholm D, et al. Cognitive therapy for bipolar illness - a pilot study of relapse prevention. Cogn Ther Res 2000; 24: 503-20.

50 Lam DH, Watkins ER, Hayward P, Bright J, Wright K, Kerr N, et al. A randomized controlled study of cognitive therapy for relapse prevention for bipolar affective disorder: outcome of the first year. Arch Gen Psychiatry 2003; 60: 145-52.

51 Mohr DC, spring B, Freedland KE, Beckner V, Arean P, Hollon SD, et al. The selection and design of control conditions for randomized controlled trials of psychological interventions. Psychother Psychosom 2009; 78: 275-84.

52 Lauder S, Chester A, Castle D, Dodd S, Gliddon E, Berk L, et al. A randomized head to head trial of MoodSwings.net.au: an internet based self-help program for bipolar disorder. J Affect Disord 2015; 171: 13-21.

53 Lin $\mathrm{C}-\mathrm{J}$, Huang $\mathrm{Y}-\mathrm{H}$, Huang $\mathrm{K}-\mathrm{Y}$, et al. MAPS group therapy model for bipolar disorder (NCT01152034). ClinicalTrials.gov, 2015.

54 Madigan K, Egan P, Brennan D, Hill S, Maguire B, Horgan F, et al. A randomised controlled trial of carer-focussed multi-family group psychoeducation in bipolar disorder. Eur Psychiatry 2012; 27: 281-4

55 Meyer TD, Hautzinger M. Cognitive behaviour therapy and supportive therapy for bipolar disorders: relapse rates for treatment period and 2-year follow-up. Psychol Med 2012; 42: 1429-39.

56 Miklowitz DJ, Simoneau TL, George EL, Richards JA, Kalbag A, Sachs-Ericsson $\mathrm{N}$, et al. Family-focused treatment of bipolar disorder: 1-year effects of a psychoeducational program in conjunction with pharmacotherapy. Biol Psychiatry 2000; 48: 582-92.

57 Miklowitz DJ, George EL, Richards JA, Simoneau TL, Suddath RL. A randomized study of family-focused psychoeducation and pharmacotherapy in the outpatient management of bipolar disorder. Arch Gen Psychiatry 2003; 60: $904-12$

58 Miller IW, Solomon DA, Ryan CE, Keitner GI. Does adjunctive family therapy enhance recovery from bipolar I mood episodes? J Affect Disord 2004; 82: 431-6.

59 Perich T, Manicavasagar V, Mitchell PB, Ball JR, Hadzi-Pavlovic D. A randomized controlled trial of mindfulness-based cognitive therapy for bipolar disorder. Acta Psychiatr Scand 2013; 127: 333-43.

60 Perlick DA, Miklowitz DJ, Lopez N, Chou J, Kalvin C, Adzhiashvili V, et al Family-focused treatment for caregivers of patients with bipolar disorder. Bipolar Disord 2010; 12: 627-37.

61 Perry A, Tarrier N, Morriss R, McCarthy E, Limb K. Randomised controlled trial of efficacy of teaching patients with bipolar disorder to identify early symptoms of relapse and obtain treatment. BMJ 1999; 318: 149-53.

62 Rea MM, Tompson MC, Miklowitz DJ, Goldstein MJ, Hwang S, Mintz J. Familyfocused treatment versus individual treatment for bipolar disorder: results of a randomized clinical trial. J Consult Clin Psychol 2003; 71: 482-92.

63 Reinares M, Colom F, Sanchez-Moreno J, Torrent C, Martinez-Aran A, Comes $\mathrm{M}$, et al. Impact of caregiver group psychoeducation on the course and outcome of bipolar patients in remission: a randomized controlled trial. Bipolar Disord 2008; 10: 511-9.

64 Sajatovic M, Davies MA, Ganocy SJ, Bauer MS, Cassidy KA, Hays RW, et al. A comparison of the life goals program and treatment as usual for individuals with bipolar disorder. Psychiatr Serv 2009; 60: 1182-9.

65 Schmitz JM, Averill P, Sayre S, Mccleary P, Moeller FG, Swann A. Cognitivebehavioral treatment of bipolar disorder and substance abuse: a preliminary randomized study. Addict Disord Their Treat 2002; 1: 17-24.

66 Scott J, Garland A, Moorhead S. A pilot study of cognitive therapy in bipolar disorders. Psychol Med 2001; 31: 459-67.

67 Scott J, Paykel E, Morriss R, Bentall R, Kinderman P, Johnson T, et al. Cognitive-behavioural therapy for severe and recurrent bipolar disorders: randomised controlled trial. Br J Psychiatry 2006; 188: 313-20.

68 Simon GE, Ludman EJ, Unutzer J, Bauer MS, Operskalski B, Rutter C. Randomized trial of a population-based care program for people with bipolar disorder. Psychol Med 2005; 35: 13-24.

69 Smith DJ, Griffiths E, Poole R, di Florio A, Barnes E, Kelly MJ, et al. Beating bipolar: exploratory trial of a novel Internet-based psychoeducational treatment for bipolar disorder. Bipolar Disord 2011; 13: 571-7. 
70 Van Dijk S, Jeffrey J, Katz MR. A randomized, controlled, pilot study of dialectical behavior therapy skills in a psychoeducational group for individuals with bipolar disorder. J Affect Disord 2013; 145: 386-93.

71 Van Gent EM, Zwart FM. Psychoeducation of partners of bipolar-manic patients. J Affect Disord 1991; 21: 15-8.

72 Weiss RD, Griffin ML, Jaffee WB, Bender RE, Graff FS, Gallop RJ, et al. A 'community-friendly' version of integrated group therapy for patients with bipolar disorder and substance dependence: a randomized controlled trial. Drug Alc Depend 2009; 104: 212-9.

73 Williams JMG, Alatiq Y, Crane C, Barnhofer T, Fennell MJ, Duggan DS, et al Mindfulness-based cognitive therapy (MBCT) in bipolar disorder: preliminary evaluation of immediate effects on between-episode functioning I Affect Disord 2008; 107: 275-9.

74 Zaretsky A, Lancee W, Miller C, Harris A, Parikh SV. Is cognitive-behavioural therapy more effective than psychoeducation in bipolar disorder? Can J Psychiatry 2008; 53: 441-8.

75 Swartz HA, Frank E, Cheng Y. A randomized pilot study of psychotherapy and quetiapine for the acute treatment of bipolar II depression. Bipolar Disord 2012; 14: 211-6.

76 Friedberg JP, Lipsitz SR, Natarajan S. Challenges and recommendations for blinding in behavioral interventions illustrated using a case study of a behavioral intervention to lower blood pressure. Patient Educ Couns 2010; 78: $5-11$.
77 Popp L, Schneider S. Attention placebo control in randomized controlled trials of psychosocial interventions: theory and practice. Trials 2015; 16: 150.

78 Cuijpers $P$, Van Straten A, Warmerdam L, Smits N. Characteristics of effective psychological treatments of depression: a metaregression analysis. Psychother Res 2008; 18: 225-36.

79 Otto MW, Simon NM, Wisniewski SR, Miklowitz DJ, Kogan JN Reilly-Harrington NA, et al. Prospective 12-month course of bipola disorder in out-patients with and without comorbid anxiety disorders. Br J Psychiatry 2006; 189: 20-5.

80 Perlis RH, Ostacher MJ, Patel JK, Marangell LB, Zhang H, Wisniewski SR, et al. Predictors of recurrence in bipolar disorder: primary outcomes from the Systematic Treatment Enhancement Program for Bipolar Disorder (STEP-BD). Am J Psychiatry 2006; 163: 217-24.

81 Mitchell PB. Bipolar disorder and anxiety: a comorbidity needing better treatments. Lancet Psychiatry 2015; 2: 671-2.

82 Shinohara $\mathrm{K}$, Honyashiki M, Imai H, Hunot V, Caldwell DM, Davies $\mathrm{P}$, et al. Behavioural therapies versus other psychological therapies for depression. Cochrane Database Syst Rev 2013; 10: CD008696.

83 Lam DH, Hayward P, Watkins ER, Wright K, Sham P. Relapse prevention in patients with bipolar disorder: cognitive therapy outcome after 2 years. Am J Psychiatry 2005; 162: 324-9.

\section{psychiatry} in literature

\section{Medea: a mythological case of familial serial killer?}

\author{
Philippe Charlier, Saudamini Deo, Michel De Grèce and Anne-Sophie Wecker
}

In Greek mythology, Medea is the wife of the hero Jason and originated from Cholcide (in today's Georgia). She is a sorceress associated with the cult of Hecate, goddess of witchcraft, poisons and ghosts. In Seneca's play she kills her younger brother Apsyrtus and cuts him into pieces that she then scatters in the sea. When her husband divorces her for a younger woman, Creusa, Medea presents her with a poisonous burning dress, kills Creusa's father Creon, then sets fire to their palace in Corinth, and finally, she bleeds to death the two children she had with Jason, Mermeros and Pheres. Back in her homeland, she kills her uncle Perses to reinstate her father Aeëtes to the throne. Medea's murderous count totals six homicides, almost exclusively within the family circle. A final, seventh homicide could be added to this list, when Medea encourages the daughters of King Pelias to kill, dismember and then cook their father.

Medea fits the definition of a serial killer, in the sense that she has killed at least three people in different locations and at different points in time. Her character seems clearly psychopathological. She derives satisfaction from killing ('I rejoice, I rejoice', v. 912; 'I feel rising in me, against me, a great enjoyment', v. 992-3). She is described as in a state of 'trance' ('furore') before the act, a state comparable or similar to depersonalisation. Some of these killings appear to have sexual aspects: for example, the murder of her two children is described in terms suggestive of an orgasmic component (v. 991-2) and pleasure (v. 1016-7). She also wants her infamy to outlive her, transforming the murders into a homicidal performance by killing her second son on the roof and showing the corpse of the first son to the crowd of Corinth (v. 976-7). At times, she seems to lose contact with reality, by not recognising and acknowledging Jason's children as her own. And, by constantly reminding herself of her past murders, she gains courage and stimulation to commit new ones.

Medea is the granddaughter of the Sun and hence is symbolically dominated by fire, an element whose preponderance in the body, according to Hippocratic medicine, is a causal factor for yellow bile and a particularly angry mood. How, then, was she to evade this kind of 'genetic' predisposition?

The mythological character of Medea is well known to psychologists and psychiatrists as the 'Medea complex' described as the killing of one's own children in revenge against an abandoning husband. Perhaps, one could add another dimension to Medea's character - that of a serial killer. 